\title{
Photopleochroism of surface-barrier structures based on semiinsulating cadmium telluride
}

\author{
G.A. Ilchuk
}

\begin{abstract}
The method of mass-transfer reaction with compounds $\mathrm{NH}_{4}(\mathrm{Cl}, \mathrm{Br}, \mathrm{J})$ as a transporting agent is developed, and single crystals of semiinsulating $\mathrm{CdTe}$ are grown. Interaction of plane-polarized radiation with In- $p$-CdTe barrier fabricated on these single crystals is investigated.

It is shown that at incidence of plane-polarized radiation at incidence angle $\Theta>0^{\circ}$ upon a surface of barrier the induced photopleochroism appears and it is proportional to the angle squared. The conclusions about the high quality both of the single crystals obtained by chemical transport reactions and of the interface of the structures as well as about the possibilities for using such structures as polarimetric analyzers of radiation are made.
\end{abstract}

Keywords: cadmium telluride, doping, Shottky barrier, linear-polarized radiation, photopleochroism, polarimetric analyzer.

Paper received 05.04.00; revised manuscript received 30.05.00; accepted for publication 06.06.00.

\section{Introduction}

Nowadays, a new wave of interest is observed to obtaining semiinsulating CdTe single crystals doped by chlorine. This is due to high sensitivity of X-ray and $\gamma$-radiation detectors fabricated on those crystals [1]. We have studied the processes of CdTe growing by using the chemical mass-transfer reactions (CTR) method with $\mathrm{NH}_{4}(\mathrm{Cl}$, $\mathrm{Br}, \mathrm{J})$ compounds as a tranporting agent [2]. It has been revealed that such growing processes are accompanied by doping of $\mathrm{CdTe}$ with galogens $\mathrm{Cl}, \mathrm{Br}, \mathrm{J}$ and permit one to obtain semiinsulating materials. Within the framework of complex investigations of the optoelectronic properties of the grown $\mathrm{CdTe}\langle\mathrm{VII}\rangle$ single crystals, we have studied the peculiarities of interaction of the linearly polarized radiation with Shottky barriers formed on these crystals.

\section{Samples and methods of measurements}

We have studied the seminsulating CdTe single crystals grown by the CTR method. The crystals had a form of parallellepipeds about $0.5 \times 1.0 \times 5.0 \mathrm{~mm}^{3}$ in size. In some cases a laser stimulation of the growth was performed. Electrical parameters of these single crystals were measured in weak electric and magnetic fields. According to our measurements of Hall effect and thermal EMF, the charge carrier transport is performed by holes whose con- centration and mobility attained $10^{8} \ldots 10^{10} \mathrm{~cm}^{-3}$, and $50 \mathrm{~cm}^{2} /(\mathrm{V} \cdot \mathrm{c})$, respectively.

Electric properties of the grown crystals were close for all transporting agents used. Spectral distribution of photoluminescence $(T=77 \mathrm{~K})$ and photovoltaic effect $(T=300 \mathrm{~K})$ revealed certain galogen-specific peculiarities that made it possible to conclude that the grown crystals were doped with galogens $(\mathrm{Cl}, \mathrm{Br}, \mathrm{J})$. Surface barrier In- $p$-CdTe structures were fabricated by thermal evaporation in vacuum ( $p \approx 10^{-5}$ Torr) of the indium layer $(d=1 \mu \mathrm{m})$. The layer was deposited upon mirror smooth growth surfaces of the CdTe single crystal. Chemically precipitated Au layers were used as ohmic contacts. The structures were not thermally treated neither during formation of the contacts nor later. The SPM-2 monochromator with the $\mathrm{SiO}_{2}$ prism was used for the photoelectric measurements.

\section{Experimental results and their analysis}

Interaction of a linearly polarized radiation (LPR) with surface barrier In- $p$-CdTe structures has been studied. Photovoltaic current was measured in a short circuit regime as a function of the LPR electric field vector $E$ orientation and of the incidence angle $\Theta$.

Under irradiation of the barriers normally to the surface of a barrier contact $\left(\Theta=0^{\circ}\right)$, the photocurrent is 


\section{G.A. Ilchuk. Photopleochroism of surface-barrier structures based of ...}

practically constant over the whole sensitivity range at any orientation of the vector $E$ of incident electromagnetic wave with respect to the incidence plane.

Variation of the photocurrent with the LPR incidence angle was studied for two perpendicular space orientations of the vector $E$ (for $p$ - and $s$ - light polarizations): 1 ) when the vector $E$ of the light wave lies in the incidence plane (IP) of the ray ( $E \|$ IP) $\left.-I^{P} ; 2\right)$ when the vector $E$ of the light wave is perpendicular to the IP $(E \perp \mathrm{IP})-I^{S}$ (Fig. 1, curves 1 and 2, respectively).

The presented results of measurements of the LPRinitiated photocurrent variation with the angle of incidence of the LPR upon a In-CdTe $\langle\mathrm{VII}\rangle$ barrier, produced at different crystallographic planes of the obtained crystals, show that the photocurrent depends on orientation of the light polarization plane. This effect is known as a photopleochroism phenomenon [3, 4].

Fig. 1 shows that, for $p$-polarization, the photocurrent $I^{p}$ initially increases with increasing LPR incidence angle, has a maximum in the vicinity of $\Theta=60^{\circ}$, and begins to decrease at $\Theta>60^{\circ}$. At the same time, the photocurrent $I^{s}$ decreases monotonically from the zero point, as soon as $\Theta$ exceeds $0^{\circ}$. Such a behavior is in agreement with the analysis of the optical processes based on Fresnel $[4,5]$ formulae at oblique incidence of the LPR upon the airCdTe interface and may serve as a demonstration of high structural perfection of the grown crystals. In the abovementioned model, an increase in the photocurrent indicates a removal of the reflection losses for a $p$-polarized light, which are minimal in the vicinity of a pseudoBrewster angle $\Theta \approx 60^{\circ}$. The obtained value of the ratio $I^{p}{ }_{\Theta Б p} / I^{p} \Theta=0$ in the structures studied is usually about 1.2-

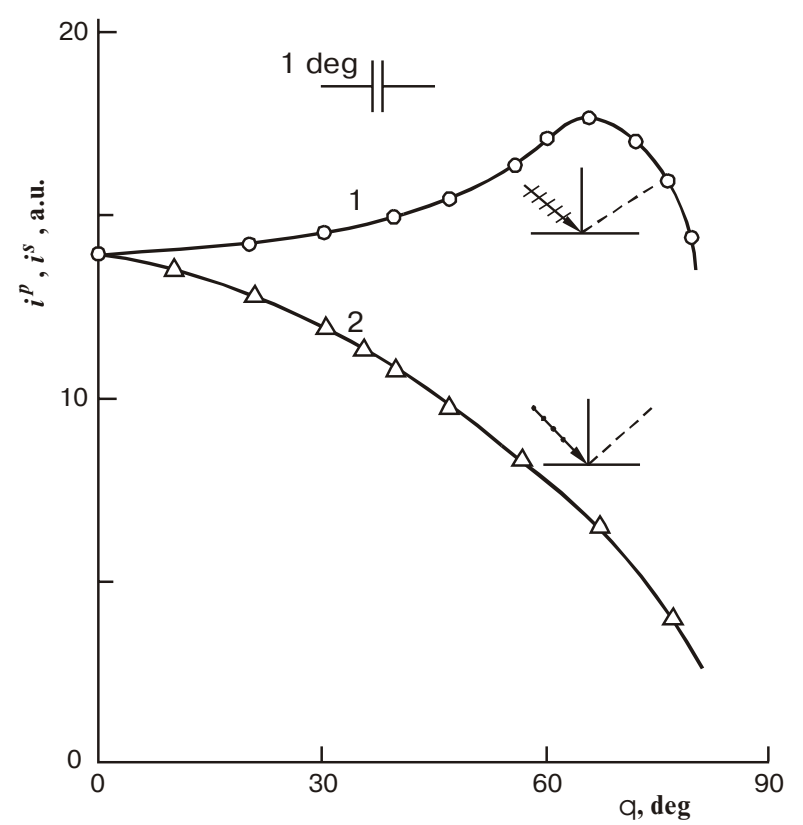

Fig. 1. Angle dependence of the short circuit photocurrent in In$p$-CdTe surface barrier structure $(T=300 K)$ for $p$ - (curve 1$)$ and $s$ - polarizations (curve 2 ) of the light, induced by linearly polarized monochromatic $(\hbar \omega=1,48 \mathrm{eV})$ radiation. The CdTe crystal is obtained by the CTR method with $\mathrm{NH}_{4} \mathrm{Cl}$ as a transporting agent.
1.3 and agrees with the effect of reflection losses decrease. As quantitative characteristics of photopleochroism, the coefficient $P_{i}$, is usually used. It is defined via the currents $I^{p}$ and $I^{s}$ of the two orthogonal polarizations of the incident beam:

$P_{1}=\left(\frac{\mu^{P}-i^{S}}{\mu^{P}+i^{S}}\right) \cdot 100 \%$.

Dependence of the induced photopleochroism coefficient $P_{i}$ and of $\sqrt{P_{i}}$ on the incidence angle $\Theta$ is given in Fig. 2.

A linear character of the experimental dependence $\sqrt{P_{i}}=f(\Theta)$ is indicative of a quadratic dependence of the photo-pleochroism coefficient. Let us emphasize the following characteristic feature of the dependence under discussion: the curve of the induced photopleochroism rises from the zero point. This is due to isotropic character of the photoactive absorption in $\mathrm{CdTe}$ at $\Theta=0^{\circ}$ and, in the case when the LPR incidence angle deviates from $0^{\circ}$, a quadratically increase of the induced photopleochroism $P_{i} \sim \Theta^{2}$ arises (Fig. 2, curve 2). This feature is in agreement with the analysis made in [6]. At incidence angle $\Theta=80^{\circ}$, the values of $P_{i}$ are close to $55-58 \%$ for structures fabricated from $\mathrm{CdTe}\langle\mathrm{VII}\rangle$ crystals. Our estimation of the refraction index of CdTe at these values of the photopleochroism coefficient gives $n \approx 2.8$. This value agrees well with the known value of this parameter in CdTe [7].

A typical dependence of spectral distribution of the induced photopleochroism coefficient for one of the surface barrier structures, that is In- $p-\mathrm{CdTe}\langle\mathrm{VII}\rangle$, is presented in Fig.3. At a fixed incidence angle $(\Theta=$ const $)$ the in-

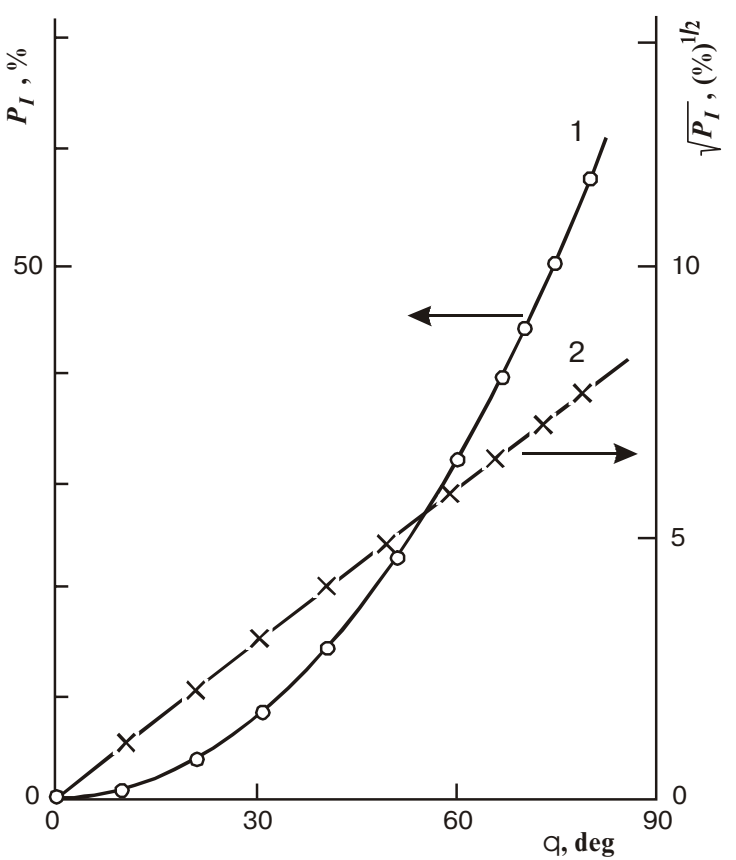

Fig. 2. Dependence of the induced photopleochroism coefficient (curves 1,2 ) on the incidence angle of linearly polarized radiation upon the surface of the In-CdTe〈VII $\rangle$ structure $(T=300 K)$ for photons with energy $1,48 \mathrm{eV}$. The CdTe crystal is grown by the CTR method with $\mathrm{NH}_{4} \mathrm{Cl}$ as a transporting agent. 


\section{G.A. Ilchuk. Photopleochroism of surface-barrier structures based of ...}

Table.Photosensitivity parameters of surface barrier structures at $300 \mathrm{~K}$ with respect to LPR

\begin{tabular}{|c|c|c|c|}
\hline Transporting agent & $\begin{array}{c}\text { Position of photosensitivity } \\
\text { maximum } \\
\hbar \omega_{m}, \mathrm{eV}\end{array}$ & $\begin{array}{c}\text { Voltage sensitivity } \\
\qquad S_{b}, \mathrm{~V} / \mathrm{W}\end{array}$ & $\begin{array}{c}\text { Azimuthal } \\
\text { photosensitivity } \\
\Phi_{b}{ }^{m}, \mathrm{~V} / \mathrm{W} \cdot \mathrm{deg}\end{array}$ \\
\hline $\mathrm{NH}_{4} \mathrm{Cl}$ & 1.475 & $9 \cdot 10^{4}$ & $1 \cdot 10^{5}$ \\
\hline $\mathrm{NH}_{4} \mathrm{Br}$ & 1.45 & $1 \cdot 10^{5}$ & $1.2 \cdot 10^{5}$ \\
\hline $\mathrm{NH}_{4} \mathrm{~J}$ & 1.36 & $6 \cdot 10^{3}$ & $7 \cdot 10^{3}$ \\
\hline
\end{tabular}

duced photopleochroism in those structures is practically unchanged over the whole photosensitivity range. The present results of the studies of the interaction between the LPR and surface barrier In- $p$-CdTe〈VII $\rangle$ structure suggest that this structure can be used as a polarimetric detector.

Rotation of a polarimetric sensor around the axis of the incident radiation beam yields azimuthal distribution of the photocurrent $i(\varphi)$. As a main parameter of the polarimetric detector, its azimuthal photosensitivity $\Phi_{b}$, is used. Its value is characterized by a change of the photovoltage produced by a unit power of the incident radiation at rotation of the $E$ vector by one degree. Its maximal value is given by [3]:

$\Phi_{b}{ }^{m}=2 S_{6} P_{I}$,

where $S_{6}$ is the voltage photosensitivity.

As one can see, at a fixed value of the incidence angle $(\Theta=$ const $)$, the maximal value of the polarization photosensitivity is determined by the maximal value of the quantum efficiency of the photo-transformation $\eta$ of the In- $p$-CdTe $\langle\mathrm{VII}\rangle$ structure, since the $P_{i}$ has a non-selective character.

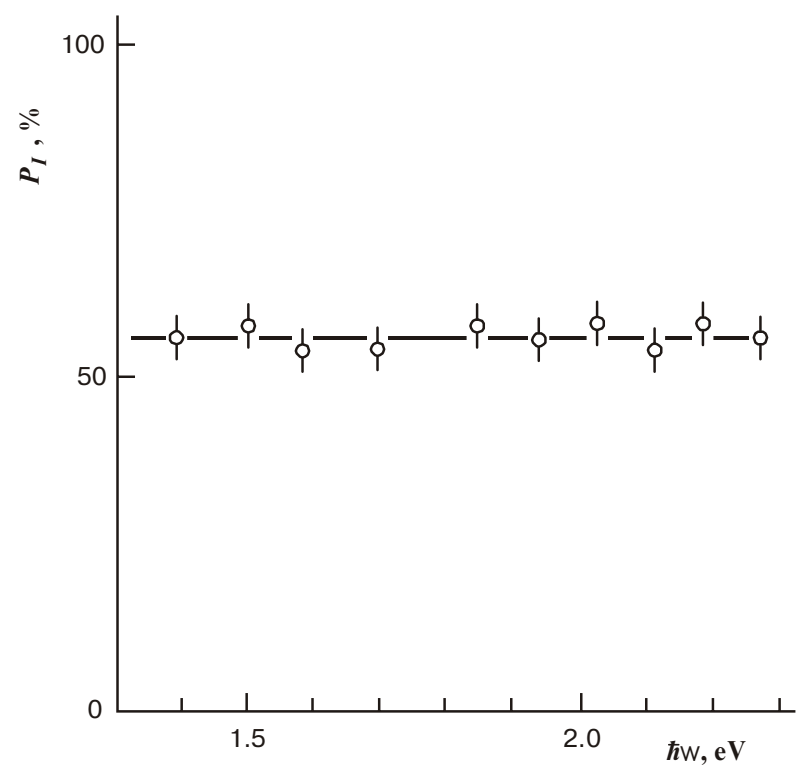

Fig. 3. Spectral dependence of the induced photopleochroism coefficient of the In-p-CdTe structure at $T=300 \mathrm{~K}\left(\Theta=80^{\circ}\right)$. The CdTe crystal is grown by the CTR method with $\mathrm{NH}_{4} \mathrm{Cl}$ as a transporting agent without activating laser irradiation.
Results of experimental measurements of the maximal value of azimuthal photosensitivity $\Phi_{B}{ }^{m}$ and voltage sensitivity $S_{b}$ of the surface barrier structures In- $p$-CdTe $\langle\mathrm{VII}\rangle$ are presented in Table.

\section{Conclusions}

Experimentally obtained agreement of the dependences of the short-circuit photocurrents, induced in a structure with the In- $p$-CdTe Shottky barrier, on the incidence angle of the beam and orientation ( $E \|$ IP or $E \perp$ IP) of its electric field vector with the dependences of the polarized radiation intensity, described by Fresnel formulae, are indicative of a high perfection of the CdTe single crystals grown by the CTR method as well as of a high quality of the metal-CdTe interface formed. The values of $S_{B}$ and $\Phi_{e}^{m}$ obtained in these experiments suggest that the surface barrier structures under investigation can be used as highly sensitive wide-range photoanalyzers of the LPR, that may serve as an analyzing device and signal sensor. Regime of photoregistration can be easily changed from a regular $\left(\Theta=0^{\circ}\right)$ to the polarimetric one $\left(\Theta \neq 0^{\circ}\right)$. On the base of the semiinsulating properties of CdTe single crystals grown by the CTR method and doped with galogens $(\mathrm{Cl}, \mathrm{Br}, \mathrm{J})$ during their growing, and taking in mind the existing practice of using such materials for registration of $\gamma$-radiation, we can predict that In- $p$ CdTe structures can be used for fabrication of analyzers of $\gamma$-radiation polarization.

Author expresses his gratitude to Professor V.I. Ivanov-Omsky for discussing the results of these studies and critical remarks.

\section{References}

1. T.Kunz, M.Zaasch, J.Meinhardt, K.W.Benz. // J.Crystal Growth 184/185, pp. 1005-1009 (1998).

2. G.A.Ilchuk, I.F.Viblyi, V.O.Dol'nikov, N.A.Ukrainets. // EMRS'98 Spring Meeting, Strasbourg (1997).

3. F.P. Kesamanly, V.Yu. Rud', Yu.V. Rud' // Fiz. Tekhnica Poluprovodn. 30 (11), pp. $1921-1942$ (1996).

4. R. Azzam, N. Bashara, Ellipsometry and polarized light (in Russian). Moscow, Mir, 1981.

5. G.S. Landsberg, Optika, Vysshaya shkola, Moscow, 1976 (in Russian).

6. G.A.Medvedkin, Yu.V.Rud. Phys. Status Solidi (a) 67, p.333 (1981).

7. Physico-chemical properties of semiconductor substancies, The handbook (in Russian). Moscow, Nauka, 1978. 